\title{
THE BIG PICARD THEOREM FOR POLYANALYTIC FUNCTIONS
}

\author{
W. BOSCH AND P. KRAJKIEWICZ
}

\begin{abstract}
ABSTRACr. Let $f, g$, and $h$ be polyanalytic in an annular neighborhood $A$ of a complex number $z_{0}$, finite or infinite, such that $g$ and $h$ do not have an essential singularity at $z_{0}$ and $g-h$ is not identically zero on $A$. It is shown that if $f-g$ and $f-h$ never vanish on $A$, then $z_{0}$ is not an essential singularity of $f$.
\end{abstract}

Let $G$ be an arbitrary region of the finite complex plane $\Gamma$. The function $f: G \rightarrow \Gamma$ is said to be polyanalytic on $G$ if and only if there exist $(n+1) \geqq 1$ functions $f_{k}$ analytic on $G$ for $k=0,1, \cdots, n$ such that

$$
f(z)=\sum_{k=0}^{n} \bar{z}^{k} f_{k}(z)
$$

for all $z \in G$, where $\bar{z}$ denotes the complex conjugate of $z$. This function is called polyentire if and only if $G=\Gamma$. Note that the functions $f_{k}$ in equation (1) are uniquely determined on $G$. Now let $z_{0}$ be a complex number, finite or infinite. Then $z_{0}$ is said to be an isolated singularity of this function $f$ if and only if there is some neighborhood $N$ of $z_{0}$ such that $N-\left\{z_{0}\right\} \subseteq G$. The point $z_{0}$ is termed an essential isolated singularity of $f$ or simply an essential singularity of $f$ if and only if $z_{0}$ is an isolated singularity of $f$ and $z_{0}$ is an essential isolated singularity of at least one of the functions $f_{k}$.

In [1], M. B. Balk derived Picard's big theorem for polyentire functions by utilizing a theorem of Saxer [4], which generalizes the classical Schottky theorem. This result of Balk cited above, leads one to speculate that Picard's big theorem may be valid for polyanalytic functions at an arbitrary isolated singularity, finite or infinite. In this paper, we establish a general version of Picard's big theorem for polyanalytic functions by means of the Poisson-Jensen integral formula [2, p. 1] and quasi-normal families [3]. As a corollary, we obtain Picard's big theorem for quotients of polyanalytic functions which satisfy certain conditions.

In order to abridge notation, we shall assume that $n$ is a fixed nonnegative integer and that $k$ is an index where $k=0,1, \cdots, n$. Next if

Received by the editors October 9, 1969.

AMS subject classifications. Primary 3081 ; Secondary 3002, 3061.

Key words and phrases. Picard's big theorem, polyanalytic functions, essential isolated singularity, Poisson-Jensen integral formula, quasi-normal families. 
$f$ is polyanalytic in $R<|z|<+\infty$ and is given by equation (1), then for $\rho \geqq 0$ it is convenient to introduce the auxiliary function $f(, \rho)$ defined by the condition that $f(z, \rho)=\sum \rho^{2 k} f_{k}(z) / z^{k}$ for $|z|>R$. Note that $f(, \rho)$ is analytic in $R<|z|<+\infty$ and that $f(z, \rho)=f(z)$ for $|z|=\rho>R$. Finally, if $f$ is an arbitrary function continuous and not zero on $|z|=\rho>0$, let $\Delta_{\rho} f$ denote $1 / 2 \pi$ times the change in the argument of $f$ around the positively oriented circumference $|z|=\rho$.

We now need some preliminary lemmas.

Lemma 1. Suppose that $f$ is polyanalytic in $R<|z|<+\infty$ and that there is some $\lambda>0$ and some sequence $\rho_{m}>R / \lambda$ diverging to $+\infty$ such that $|f(\rho z, \rho)| \leqq K \rho^{s}$ for $|z|=\lambda$ and $\rho=\rho_{m}$, where $K$ is some positive constant and $s$ is some nonnegative integer. Then $z_{0}=\infty$ is not an essential singularity of $f$.

Proof. If $f$ is given by equation (1), let $f_{k}(z)=\sum a_{\mu}^{\boldsymbol{k}} z^{\mu}$ for $R<|z|$ $<+\infty$ be the Laurent series expansion of $f_{k}$ with center $z_{0}=\infty$. Then the coefficient $b_{\mu}$ of $z^{\mu}$ in the Laurent series expansion of $f(, \rho)$ with center $z_{0}=\infty$ is $b_{\mu}=\sum \rho^{2 k} a_{\mu+\boldsymbol{k}}^{\boldsymbol{k}}$. Hence by Cauchy's inequalities we obtain the estimate $\left|\sum \rho^{\mu+2 k-s} a_{\mu+k}^{k}\right| \leqq K / \lambda^{\mu}$, which is valid for $\rho=\rho_{m}$. In the above inequality, we see that the coefficients of positive powers of $\rho$ must vanish. Thus $a_{\nu}^{\boldsymbol{k}}=0$ whenever $\nu>s-k$. Thus $z_{0}=\infty$ is not an essential singularity of the functions $f_{k}$. From this the conclusion follows.

Lemma 2. Suppose that $f$ is polyanalytic in $R<|z|<+\infty$ and that there is some $0<\lambda \leqq 1$ and some sequence $\rho_{m}>R / \lambda$ diverging to $+\infty$ and some positive constant $K$ such that $|f(\rho z, \rho)| \geqq K$ for $|z|=\lambda$ and $\rho=\rho_{m}$. Suppose also that the sequence of integers $\Delta_{\lambda_{\rho}} f(, \rho)$ for $\rho=\rho_{m}$ is bounded above. Then $z_{0}=\infty$ is not an essential isolated singularity of $f$.

Proof. We shall first consider the case when $f$ is polyentire. Suppose that $f$ is given by equation (1). Let $g$ be the polyentire function defined by the condition that $g(z)=z^{n} f(z)$ for $z \in \Gamma$. Note that $g(z, \rho)$ $=z^{n} f(z, \rho)$ for $\rho \geqq 0$ and $|z|>0$. Evidently $g$ satisfies the hypotheses of Lemma 2. There is no loss of generality in assuming that $|g(\rho z, \rho)|$ $\geqq 1$ for $|z|=\lambda$ and $\rho=\rho_{m}$. There is some integer $p$ such that $\Delta_{\lambda \rho} g(, \rho)$ $\leqq p$ for $\rho=\rho_{m}$. Note that $g(, \rho)$ is an entire function for all $\rho \geqq 0$. Now by Jensen's integral formula $[2$, p. 3 ] we have that

$$
\begin{aligned}
& \frac{1}{2 \pi} \int_{0}^{2 \pi} \log \mid g\left(\lambda \rho e^{i \phi}, \rho\right) \mid d \phi \\
&=\sum_{\mu=1}^{q} \log \frac{\lambda \rho}{\left|a_{\mu}\right|}+\log \frac{\left|g^{(s)}(0, \rho)\right|}{s !}+s \log \lambda \rho
\end{aligned}
$$


for $\rho=\rho_{m}$, where $a_{1}, \cdots, a_{q}$ are the roots of $g(, \rho)$ in $0<|z|<\lambda \rho$ with due regard for multiplicities and $s \geqq 0$ is the multiplicity of the root $z=0$ of $g(, \rho)$. In order to estimate the right-hand side of equation (2), we make some simple observations. First there is some $R_{0}>R / \lambda$ such that $s$ is independent of $\rho$ for $\rho>R_{0}$. Thus $g^{(s)}(0, \rho)$ is a fixed polynomial in $\rho$ for $\rho>R_{0}$. Also $q=\Delta_{\lambda \rho} g(, \rho)-s \leqq p-s$ for $\rho=\rho_{m}>R_{0}$. Finally there is some $\sigma>0$ and some nonnegative integer $t$ and some $R_{1}>R_{0}+1$ such that $\left|a_{1}\right|, \cdots,\left|a_{q}\right|>\sigma / \rho^{t}$ for $\rho>R_{1}$. This last observation follows from elementary estimates on $g(, \rho)$ and the fact that $z=0$ is a root of multiplicity $s$ of $g(, \rho)$ for $\rho>R_{0}$. Now from equation (2) and the above observations, we see that there is some positive constant $K$ and some positive integer $\mu$ such that

$$
\frac{1}{2 \pi} \int_{0}^{2 \pi} \log \left|g\left(\lambda \rho e^{i \phi}, \rho\right)\right| d \phi \leqq \log K \rho^{\mu},
$$

for all $\rho=\rho_{m}>R_{1}$. From the above inequality, we may now obtain an upper estimate for $|g(z, \rho)|$ when $0 \leqq|z|<\lambda \rho$ and $\rho=\rho_{m}>R_{1}$. If $z=r e^{i \theta}$ where $0 \leqq r<\lambda \rho$, then by the Poisson-Jensen integral formula $[2, \mathrm{p} .1]$ we have that

$$
\begin{aligned}
\log |g(z, \rho)|= & \frac{1}{2 \pi} \int_{0}^{2 \pi} \log \left|\lg \left(\lambda \rho e^{i \phi}, \rho\right)\right| \frac{\lambda^{2} \rho^{2}-r^{2}}{\lambda^{2} \rho^{2}-2 \lambda \rho r \cos (\theta-\phi)+r^{2}} d \phi \\
& +\sum_{\mu=1}^{q} \log \left|\frac{\lambda \rho\left(z-a_{\mu}\right)}{\lambda^{2} \rho^{2}-\bar{a}_{\mu} z}\right|+s \log \frac{r}{\lambda \rho},
\end{aligned}
$$

for $\rho=\rho_{m}>R_{1}$, whenever $g(z, \rho) \neq 0$. Now $|g(z, \rho)| \geqq 1$ for $|z|=\lambda \rho$ and $\rho=\rho_{m}$. Hence from equation (4) we obtain the estimate

$$
\log |g(z, \rho)| \leqq \frac{\lambda \rho+r}{\lambda \rho-r} \frac{1}{2 \pi} \int_{0}^{2 \pi} \log \left|g\left(\lambda \rho e^{i \phi}, \rho\right)\right| d \phi,
$$

which is valid for $|z|=r<\lambda \rho$ and $\rho=\rho_{m}>R_{1}$, whenever $g(z, \rho) \neq 0$. If we now combine inequalities (3) and (5) and then set $r=\lambda \rho / 2$, we see that there is some positive constant $M$ and some positive integer $\nu$ such that $|g(\rho z, \rho)| \leqq M \rho^{\nu}$ for $|z|=\lambda / 2$ and $\rho=\rho_{m}>R_{1}$. From Lemma 1 , we see that $z_{0}=\infty$ is not an essential singularity of $g$. Thus $z_{0}=\infty$ is not an essential singularity of $f$. Consequently Lemma 2 has been established in the case that $f$ is polyentire. Next consider the case when $f$ is polyanalytic in $0<|z|<+\infty$ and $z_{0}=0$ is not an essential singularity of $f$. Then there exists some nonnegative integer $s$ and some polyentire function $g$ such that $g(z)=z^{8} f(z)$ for all $0<|z|<+\infty$. Evidently $g$ satisfies the hypotheses of Lemma 2. Thus $z_{0}=\infty$ is not an essential singularity of $g$. Hence $z_{0}=\infty$ is not an essential singular- 
ity of $f$. Consequently Lemma 2 has been established when $f$ is polyanalytic in $0<|z|<+\infty$ and $z_{0}=0$ is not an essential singularity of $f$. Finally, let us consider the general case when $f$ is polyanalytic in $R<|z|<+\infty$. Let $f_{k}(z)=\sum a_{\mu}^{k} z^{\mu}$ for $R<|z|<+\infty$ be the Laurent series expansion of $f_{k}$ with center $z_{0}=\infty$. Let $g_{k}(z)=\sum a_{\mu}^{k} z^{\mu}$ where $-n \leqq \mu<+\infty$ for $0<|z|<+\infty$. Let $g(z)=\sum \bar{z}^{k} g_{k}(z)$ for $0<|z|$ $<+\infty$. Note that $g$ is polyanalytic in $0<|z|<+\infty$ and that $z_{0}=C$ is not an essential singularity of $g$. It is easy to see that $f(\rho z, \rho)-g(\rho z, \rho)$ $\rightarrow 0$ uniformly on $|z|=\lambda$ as $\rho \rightarrow+\infty$. It therefore follows that $g$ satisfies the hypotheses of Lemma 2. Thus $z_{0}=\infty$ is not an essential singularity of $g$. Hence $z_{0}=\infty$ is not an essential singularity of $f$. This completes the proof of Lemma 2 .

Lemma 3. Suppose that $f$ is polyanalytic and not identically zero in $R<|z|<+\infty$ and that $z_{0}=\infty$ is not an essential singularity of $f$. Then there is some finite subset $F$ of $0<|z|<+\infty$ such that if $A$ is any closed and bounded subset of $0<|z|<+\infty$ which does not meet $F$, then there exist positive constants $K$ and $L$ and some nonnegative integer $t$ such that $L \rho^{t} \leqq|f(\rho z, \rho)| \leqq K \rho^{t}$ for all $z \in A$ and for all $\rho$ sufficiently large.

Proof. It suffices to note that there exist nonnegative integers $s$ and $t$ and a nonidentically zero polynomial $P(z)$ in $z$ such that $f(\rho z, \rho) / \rho^{t} \rightarrow P(z) / z^{s}$ uniformly for $z$ in any closed and bounded subset of $0<|z|<+\infty$ as $\rho \rightarrow+\infty$. If we now let $F$ denote the set of nonzero roots of $P$, then Lemma 3 readily follows.

We are now in a position to establish a general version of Picard's big theorem for polyanalytic functions.

THEOREM. Suppose that $f, g$, and $h$ are polyanalytic in an annular neighborhood $A$ of a complex number $z_{0}$, finite or infinite, such that $g$ and $h$ do not have an essential singularity at $z_{0}$ and $g-h$ is not identically zero in $A$. If $f-g$ and $f-h$ never vanish in $A$, then $z_{0}$ is not an essential singularity of $f$.

Proof. There is no loss in generality in assuming that $h \equiv 0$. We first consider the case when $z_{0}=\infty$. Let the annular neighborhood $A$ of $z_{0}=\infty$ be $R<|z|<+\infty$. Since the functions $f$ and $f-g$ never vanish in $R<|z|<+\infty$, there exist integers $r$ and $s$ such that $\Delta_{\rho} f=r$ and $\Delta_{\rho}(f-g)=s$ for all $\rho>R$. Next thereis so me $\delta>\sigma>R$ and some integers $u$ and $v$ such that $\Delta_{\sigma} f(, \rho)=u$ and $\Delta_{\sigma}[f(, \rho)-g(, \rho)]=v$ for all $\rho>\delta$. Thus the functions $f(, \rho)$ and $f(, \rho)-g(, \rho)$ have $r-u$ and $s-v$ zeros, respectively, in $\sigma<|z|<\rho$ for all $\rho>\delta$. Consequently, the functions $f(\rho z, \rho)$ and $f(\rho z, \rho)-g(\rho z, \rho)$ have at most $r-u$ and $s-v$ zeros, respectively, in $\sigma / \delta<|z|<1$ for all $\rho>\delta$. Now by Lemma 3, there is some 
$\sigma / \delta<a<b<1$ and some $\mu>\delta$ such that $L \rho^{t} \leqq|g(\rho z, \rho)| \leqq K \rho^{t}$ for $a<|z|<b$ and $\rho>\mu$, where $L$ and $K$ are some positive constants and $t$ is some nonnegative integer. Let $B$ denote the annulus $a<|z|<b$ and let $\rho_{m}>\mu$ be a sequence diverging to $+\infty$. For $m \geqq 1$, define $H_{m}$ on $B$ by the condition that $H_{m}(z)=f\left(\rho_{m} z, \rho_{m}\right) / g\left(\rho_{m} z, \rho_{m}\right)$ for $z \in B$. Let $\mathfrak{F C}$ denote the collection of these functions $H_{m}$ for $m \geqq 1$. First observe that each function $H_{m}$ is analytic in $B$. Next observe that each function $H_{m}$ assumes the value zero and one at most $r-u$ and $s-v$ times, respectively, in $B$. Consequently, the family $\mathfrak{H}$ is quasinormal of order $q$ on $B$ where $0 \leqq q \leqq \max (r-u, s-v)[3$, p. 67]. Let us first consider the case when $q \geqq 1$. Then there is some subsequence $H_{m_{v}}$ of $H_{m}$ and $q$ points $z_{1}, \cdots, z_{q}$ of $B$ such that $H_{m_{v}}(z) \rightarrow \infty$ almost uniformly on $B-\left\{z_{1}, \cdots, z_{q}\right\}$ as $v \rightarrow+\infty$. Then we may choose $a<\lambda<b$ so that $H_{m_{v}}(z) \rightarrow \infty$ uniformly on $|z|=\lambda$ as $v \rightarrow+\infty$. Hence there is some integer $v_{0}$ so that $\left|H_{m_{v}}(z)\right| \geqq 1$ for $|z|=\lambda$ and $v \geqq v_{0}$. Thus $|f(\rho z, \rho)| \geqq|g(\rho z, \rho)| \geqq L \rho^{t} \geqq L \mu^{t}$ for $|z|=\lambda$ and $\rho=\rho_{m_{v}}$ and $v \geqq v_{0}$. Note also that $\Delta_{\lambda_{\rho}} f(, \rho) \leqq \Delta_{\rho} f(, \rho)=r$ for $\rho=\rho_{m_{v}}$ and $v \geqq v_{0}$. Thus by Lemma $2, z_{0}=\infty$ is not an essential singularity of $f$. Next consider the case when $q=0$. Thus the family $\mathcal{H C}$ is normal in $B$. There are now two possibilities to consider. First, there may be a subsequence of $H_{m}$ which diverges to infinity almost uniformly on $B$. In this eventuality, one readily verifies as before that $z_{0}=\infty$ is not an essential singularity of $f$. Second, there may be a subsequence $H_{m_{v}}$ of $H_{m}$ which converges almost uniformly on $B$. Choose $a<\lambda<b$. Hence there is some positive constant $M$ and some integer $v_{0}$ so that $\left|H_{m_{v}}(z)\right| \leqq M$ for $|z|=\lambda$ and $v \geqq v_{0}$. Thus $|f(\rho z, \rho)| \leqq M|g(\rho z, \rho)|$ $\leqq M K \rho^{t}$ for $|z|=\lambda$ and $\rho=\rho_{m_{v}}$ and $v \geqq v_{0}$. Hence by Lemma 1 , we see that $z_{0}=\infty$ is not an essential singularity of $f$. Thus the theorem has been established in the case that $z_{0}=\infty$. The case when $z_{0}$ is finite is reduced to the case when $z_{0}$ is infinite by means of an inversion with center $z_{0}$. This completes the proof of the theorem.

As applications of the above theorem, we offer the following corollaries.

Corollary 1. Suppose that $f$ is polyanalytic with an isolated singularity $z_{0}$. If $f$ omits two distinct finite values in some annular neighborhood of $z_{0}$, then $z_{0}$ is not an essential isolated singularity of $f$.

COROLlary 2. Let $z_{0}$ be an essential isolated singularity of a polyanalytic function $f$. Then in every annular neighborhood of $z_{0}$, the values of the function are dense in the extended complex plane.

Corollary 3. Suppose that $f$ and $g$ are polyanalytic functions with an isolated singularity $z_{0}$ such that $z_{0}$ is not an essential singularity of 
$g$ and $g$ never vanishes in some annular neighborhood of $z_{0}$. If the function $\mathrm{f} / \mathrm{g}$ omits two distinct finite values in this annular neighborhood of $z_{0}$, then $z_{0}$ is not an essential singularity of $f$.

\section{REFERENCES}

1. M. B. Balk, On the values taken by entire polyanalytic functions, Dokl. Akad. Nauk SSSR 167 (1966), 12-15=Soviet Math. Dokl. 7 (1966), 308-311. MR 33 \#7544.

2. W. K. Hayman, Meromorphic functions, Oxford Math. Monographs, Clarendon Press, Oxford, 1964. MR 29 \#1337.

3. P. Montel, Leçons sur les familles normales de fonctions analytiques et leurs applications, Gauthier-Villars, Paris, 1927.

4. W. Saxer, Über eine Verallgemeinerung des Satzes von Schottky, Compositio Math. 1 (1934), 207-216.

University of Nebraska, LinColn, Nebraska 68508 